Article

\title{
Circular Statistics Applied to the Study of the Solar Radiation Potential of Rooftops in a Medium-Sized City
}

\author{
María-Eugenia Polo ${ }^{1, *(1)}$, Mar Pozo $^{2}$ and Elia Quirós ${ }^{2}$ (i) \\ 1 Graphic Expression Department, University of Extremadura, 06800 Mérida, Spain \\ 2 Graphic Expression Department, University of Extremadura, 10003 Cáceres, Spain; mmpozo@unex.es (M.P.); \\ equiros@unex.es (E.O.) \\ * Correspondence: mepolo@unex.es; Tel.: +34-924-673-084
}

Received: 15 September 2018; Accepted: 16 October 2018; Published: 18 October 2018

check for updates

\begin{abstract}
Solar energy constitutes one of the most effective alternative energy sources for combating climate change. However, the solar potential in a city can vary depending on the urban morphology. The purpose of this paper is to perform a directional statistical analysis of the distribution of the monthly solar potential of rooftops in the city of Cáceres, Spain, in relation to the orientations and slopes of the rooftops. Two residential areas, one in the city center and one on the outskirts of the city, and an industrial zone, all of which exhibit different urban morphologies, have been evaluated. Statistics have been assessed in consideration of the orientation and slope values of the rooftops as circular data, and the radiation values as linear data. The three dissimilar urban morphologies result in different solar potential values, and the monthly disaggregation of the data enables the ability to detect the differences existing in the solar potential between each zone, during each month. The proposed analysis could also be extrapolated to urban planning for the design of more sustainable cities to face the challenges associated with climate change.
\end{abstract}

Keywords: solar potential; solar radiation; circular data; statistical analysis; rooftop orientation

\section{Introduction}

Low-carbon electricity production using photovoltaic (PV) panels or building-integrated PVs on rooftops contributes to an increased use of renewable energies [1,2]. Among the various sources of renewable energy, the utilization of solar energy represents one of the most effective methods for combating climate change, and thus solar energy is supported by governments and policy makers worldwide [3]. Accordingly, the use of solar energy has generated the need to assess the solar potential of cities throughout developing countries [4].

Many solar potential models have been developed $[5,6]$. However, the amount of solar energy that reaches the terrestrial surface depends on the local weather conditions, atmospheric effects, surveying, orientation, and the slope and solar incident angle in the study area [7]. Additionally, the insolation of the terrestrial surface is influenced by the rotation and translation of the Earth around the Sun and fluctuates with the cloud cover [8]. Nevertheless, several works have concluded that the abovementioned models can be adjusted to accommodate small study zones [9,10]; thus, greatly increasing the number of potential studies for investigating the solar potential of cities in developed countries [11,12].

For many years, solar potential models of rooftops in urban areas have typically been generated by geographical information systems (GIS) data [7]. Recently, to improve the precision of these models, light detection and ranging (LiDAR) data have been included for urban areas that take into account the 
horizontal surfaces (rooftops) only, excluding the walls, and discard the three-dimensional analysis, thereby enabling a study with other variables such as shading, orientation, and inclination [13]. As a result, several algorithms have been developed to estimate the footprint area and shadows of buildings [14-16]. At the same time, another technique known as rooftop digitization has been implemented by cartography or ortho mapping to evaluate solar models, as in [17]. Additionally, some studies have developed a methodology to estimate the shadow factor in urban terrain considering the different conditions of the sky [18]. Currently, the most accurate solar potential model in urban areas is obtained using a digital surface model (DSM) determined from LiDAR data and local meteorological time series [19-21]. The high density of LiDAR data allows the solar potential model to consider the characteristics of the surroundings, such as the presence of vegetation, urban furniture, and shadows [21-23].

However, estimating the solar potential in urban areas can vary depending on the urban morphology [24]. Several works have demonstrated the relationship between the urban morphology and solar energy potential and its influence on the availability of solar energy [25]. Therefore, several aspects, such as the orientation, height, urban compactness, and the shapes of buildings, should be considered within the concept of the urban geometry. All of these aspects influence the shadows of the surrounding urban furniture [26,27].

If a further evaluation of the solar radiation is performed, the orientation and the slope of the rooftop, which constitute crucial issues, can be calculated from LiDAR data. Some studies have confirmed that the main factors determining the relationship between the insolation level and the building shape are the width-length ratio and the building orientation [28]. Additionally, other works have demonstrated that the solar energy potential of a rooftop depends on both its shape and its orientation [24]. Consequently, these parameters are directly associated with the urban morphology. As a result, many investigations have been performed, all around the world, on the importance of the optimal orientation of PV panels [29-31]. To perform an evaluation, vectors are defined for the azimuth, rooftop orientation, solar module, and received radiation, but the slope of the roof of a building constitutes another important factor that should be taken into account. The study of this vector set can enhance the study of the distribution of the solar potential in a city.

Circular statistics or directional statistics address angular data, axes, or vectors and enable an analysis of orientations in two dimensions. Circular statistics are applied in a variety of disciplines, such as biology [32], meteorology (wind analysis) [33], geography [34], remote sensing [35] and cartography [36], to name a few. The analysis of angular data requires special methods because directional data are different from linear data. For example, the origin of circular data is an arbitrary direction (e.g., the $\mathrm{X}$ axis or north), and operations on circular data are performed within the interval of $0^{\circ}-360^{\circ}$. Moreover, the relationships among circular data are not the same as those among linear data. For example, a value of 30 is greater than a value of 10 , but $30^{\circ}$ is not greater than $10^{\circ}$ when considering that it represents cyclical information [37]. Comprehensive works have addressed circular data [38-40].

Several studies such as $[13,25,41]$ have employed circular graphics to represent the orientations of buildings with regard to the solar potential. Furthermore, reference [41] studied the slopes of roofs and their contribution to the PV efficiency, but the experiment employed parametrically modelled buildings. Additionally, reference [42] analyzed circular histograms, representing the orientation and its corresponding solar potential, and concluded that the orientation is a crucial factor for the yearly solar irradiation. In this way, reference [13] assessed the orientation distribution of roofs as a function of the roof orientation in 538 identified buildings. However, the general tendency of the abovementioned works was to evaluate the solar potential from an annual point of view, and to model the buildings parametrically in most cases. In addition, no significant metrics dealing with directional statistics have been performed. The purpose of this paper is to perform a directional statistical analysis of the distribution of the monthly solar potential of rooftops in the city of Cáceres in relation to the orientations and slopes of the rooftops in three different neighborhoods. The added value of the 
present work is the higher precision of the data achieved as a consequence of the roofs having a greater level of detail (i.e., photogrammetrically restituted gables) and the monthly disaggregation of the results. In addition, three variables, namely the solar orientation, the roof slope, and the monthly solar radiation, in each building within the study areas are used to perform the statistical analysis, thereby enabling an analysis of how different urban structures affect the solar potential values. To carry out this analysis, different neighborhoods have been selected in terms of their street structure, building typology, and use (i.e., a central neighborhood in a downtown area, a residential area, and an industrial zone) in the study area.

Accordingly, the orientation and slope values will be treated as circular data, and the radiation values will be treated as linear data. Both the circular and the linear analyses will be performed and complemented with a study of the correlations between both variables to analyze the distribution of radiation in relation to the different urban morphologies within the city. The monthly potential solar energy reaching the rooftops will be calculated using the methodology described in [21].

\section{Study Case}

The working area is Cáceres, Spain, a medium-sized city with a mixed urban distribution and a population of approximately 100,000 (Figure 1). This city, which spans an area of $23 \mathrm{~km}^{2}$, was declared a World Heritage City by UNESCO in 1986.

Throughout its history, Cáceres has developed as a radio-concentric city, while favoring gradual growth and peripheral crowns based on residential polygons of unequal relationships and connections with each other. Currently, numerous different urban morphologies can be distinguished in the city:

- $\quad$ The Central Zone, whose origin is the historic center, which is predominated by a combination of low- to medium-density residential buildings.

- The East and West Residential Zones, which are predominated intensively by residential buildings.

- The West Single-Family Residential Zone, which is almost exclusively dominated by isolated detached and semi-detached buildings.

- The Northern Zone, which was constructed as much from urban development as from administrative reclassifications. Single-family residences coexist with a collection of medium-density residences in this area.

- The University Zone to the east, which is mainly institutional.

- The Industrial Zone, which is mainly located to the southeast and the northwest.

According to [21], Cáceres is a city with a substantial renewable energy potential due to the monthly solar potential that, in some of the buildings, exceeds $7 \mathrm{kWh} / \mathrm{m}^{2}$ during the summer months, namely May, June, and July. 


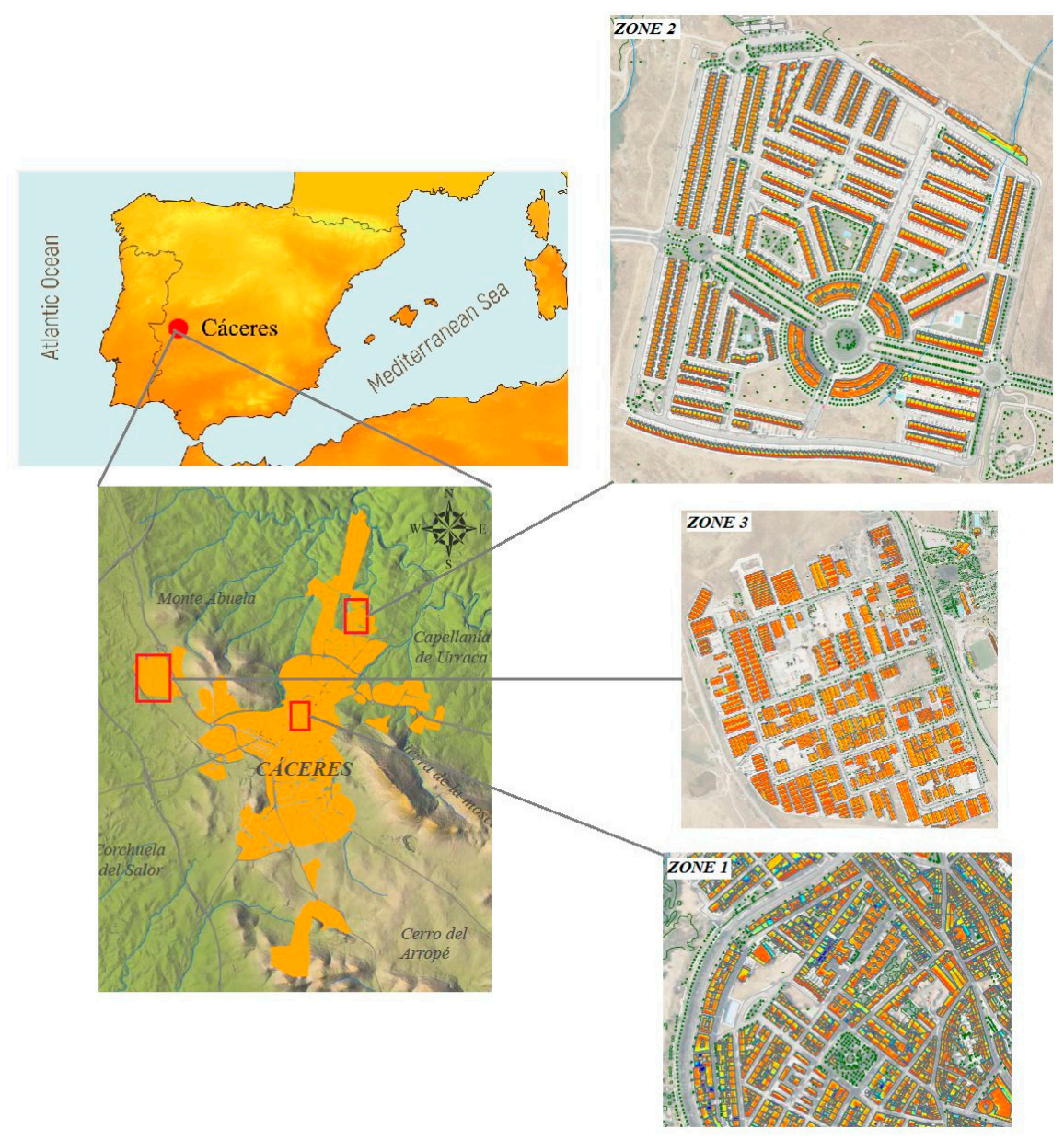

Figure 1. Working area and distribution of the three studied neighborhoods.

Three neighborhoods were selected according to their different urban characteristics (Table 1): A portion of the city center that had irregular streets and different types of buildings (zone 1), a new residential area on the outskirts of the city that had more regular and wider streets and similar types of buildings (zone 2), and an industrial area (zone 3) that had wide streets and similar types of industrial warehouse buildings.

Table 1. Main characteristics of the three study areas.

\begin{tabular}{cccc}
\hline & Zone 1 & Zone 2 & Zone 3 \\
\hline Type & $\begin{array}{c}\text { Residential area in } \\
\text { the city center }\end{array}$ & $\begin{array}{c}\text { Residential area on } \\
\text { the outskirts }\end{array}$ & $\begin{array}{c}\text { Industrial area on } \\
\text { the outskirts }\end{array}$ \\
\hline $\begin{array}{c}\text { Number of Gables } \\
\text { (Studied Polygons) }\end{array}$ & 97,424 & 83,064 & 363,898 \\
\hline Type of Buildings & $\begin{array}{c}\text { Different types } \\
\text { of buildings }\end{array}$ & Terraced family homes & Industrial warehouses \\
\hline
\end{tabular}

The rooftop delineations were provided by the local council administration, and their gables were detailed in most of the buildings (Figure 2). This cartographic information had an accuracy of $10 \mathrm{~cm}$ and represented all elements, such as the edges of rooftops, yards, cantilevers, and balconies, which can be appreciated at a scale of $1 / 500$. In addition, other significant elements, such as chimneys and antennas, which could be crucial for the estimation of solar shadows, were also included in the delineation.

The variables to be analyzed in these three cases were as follows: 
- The orientation value of the rooftops, measured as the compass direction (north) of the descending slope of the gables, measured clockwise, and given in degrees.

- The slope of the gables given in degrees and measured from the horizontal plane.

- The radiation values obtained from GIS data and a DSM of the city from airborne LiDAR data [21]. Radiation data are given in units of $\mathrm{kWh} / \mathrm{m}^{2}$. The computed solar potential model by GIS considers the orientations and tilts of the rooftops and the shadows from the surroundings, due mainly to vegetation and urban furniture, contrasting with most solar radiation recording stations which measure only the total horizontal solar intensity [9].

As an example, the rooftop distribution of zone 1, which exhibited irregular streets and different types of buildings, is shown in Figure 2.

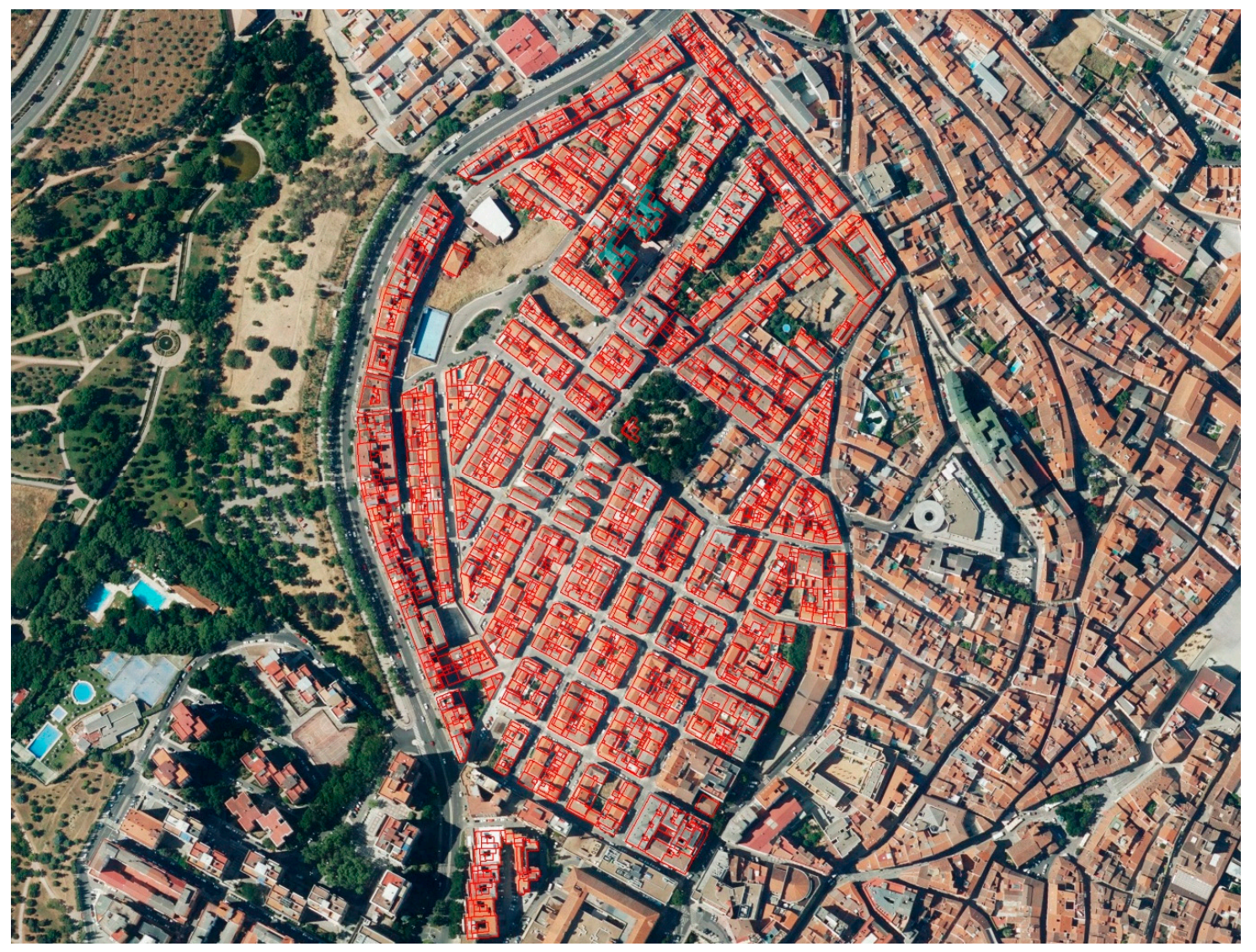

Figure 2. Vectors of the rooftops within a residential area in the city center (zone 1).

Four applications, namely Oriana, VecStatGraphs2D, IBM SPSS, and Excel, were used to perform the statistical analysis. Oriana [43] is a commercial application written for Microsoft Windows that calculates statistics and graphics for circular data, angles or directions measured in degrees, time of day, month of year, etc. VecStatGraphs2D [33] is an R package [44], designed for the statistical analysis of $2 \mathrm{D}$ vectors, that generates a set of statistics for both linear data (e.g., the arithmetic mean, standard deviation, variance, and skewness coefficient) and circular data (e.g., the mean azimuth, mean module, and circular variance), and it also generates graphics to address circular data. IBM SPSS [45] is a platform that offers advanced statistical analyses, and it is especially used in the social sciences field.

\section{Methods}

All GIS data and DSM data derived from airborne LiDAR data could be analyzed to study the solar radiation potential of the rooftops in this study. The rooftop delineation was provided by the local council administration of Cáceres for the three selected study zones. As a result, a large quantity 
of data, namely 97,424, 83,064, and 363,898 gables for zones 1, 2, and 3, respectively (see Table 1), were obtained for the three study areas. Every gable contained one orientation value, one slope value, and twelve radiation values (one for each month of the year). The orientation value was measured as the compass direction (clockwise from north) in the direction of the descending slope of the gables and was given in degrees. The slope of the gables was measured from the horizontal plane in degrees. All of the information was analyzed from different perspectives, considering that sets of vectors are created with both radiation values (the module of the vector) and orientation values (the azimuth of the vector).

The modules of the vectors (radiation values expressed in $\mathrm{kWh} / \mathrm{m}^{2}$ ) were analyzed by linear statistics. The usual analysis approach comprises descriptive statistics, such as the maximum and minimum values, arithmetic mean, median, standard deviation, skewness, kurtosis, and tests of normality for each month and zone. The arithmetic mean, minimum, and maximum values are displayed in Table 2. The numerical statistics were complemented with histograms that represented the radiation values along the $X$ axis, grouped in intervals, and the absolute frequencies of the radiation falling in each interval along the $\mathrm{Y}$ axis. One histogram was made for each month and area (36 in total) to compare the radiation distribution throughout the year in the three studied zones. The histograms of July and December for the three areas are shown in Figure 3.

The azimuths of the vectors (orientation values expressed in degrees) were analyzed by circular statistics. As circular or directional statistics address angular data, the slopes of the gables were also analyzed with the same procedure. In this case, the statistics chosen to define the distribution of the data were the mean azimuth, the circular standard deviation, the circular variance, and the concentration parameter.

The mean azimuth $(\bar{\theta})$ was the azimuth of the vector sum (R) of the data, and it was analogous to the arithmetic mean in linear data; it was computed from the sines (S) and cosines (C) of the $n$ individual angular data.

$$
\bar{\theta}=\operatorname{arctg} \frac{S}{C} ; C=\sum_{i=1}^{n} \cos \theta_{i} ; S=\sum_{i=1}^{n} \operatorname{sen} \theta_{i}
$$

The circular variance and the circular standard deviation were calculated as a measure of the dispersion of the data. The circular variance $(V)$ was calculated from the vector sum $(R)$ and the mean module $(\bar{R})$ according to the following:

$$
\bar{R}=\frac{R}{n} V=1-\bar{R}
$$

The circular standard deviation $(v)$ is similar to the standard deviation for linear data, and its expression is as follows:

$$
v=\sqrt{-2 \log (\bar{R})}
$$

The concentration parameter, also known as the von Mises parameter, is a measure of the concentration of the data around a preferred direction within the circle in the von Mises distribution, which is a symmetric unimodal distribution similar to a Gaussian distribution in linear data [37]. This parameter is 0 in a uniform distribution and is considered significant when greater than 2 . In a uniform distribution, all directions from $0^{\circ}$ to $360^{\circ}$ are equally probable, and the distribution is spread uniformly around the circle.

The radiation values (Figure 4) and the slope values (Figure 5) were plotted to analyze their distributions. It should be noted that these graphics consider the orientation and the slope values as unit vectors, and thus it will be necessary to obtain more comprehensive information.

One limitation of using circular statistics is that they are designed to address vector units, whereas it is usually necessary to work with non-unitary modules. It is possible to analyze angles and modules in an independent way, but a more complete study requires employing both the module and the azimuth of each vector. 
The joint distribution of the circular and linear data is provided in a two-variable circular histogram graphic. This graphic consists of a histogram wrapped around a circle, in which each wedge-shaped section of the histogram depicts the number of observations (radiation values) falling within that portion of the range. In this graph, each wedge spans $30^{\circ}$ (a customized parameter) of the total circular range, and the length of each wedge represents the number of observations falling within that range. Every wedge divides the radiation variable into frequency classes represented by a color ramp. Blue colors represent low radiation values, whereas red colors imply a higher level of received radiation. Figure 6 shows 36 two-variable circular histogram graphics corresponding to a graph for each month and area. These graphs enabled a comparison of not only the differences in the orientations of the gables according to each area but also the distributions of the radiation and the variations of this parameter throughout the year.

Finally, the circular-linear correlation coefficient was calculated to assess the correlations among the circular variable, the orientation, the linear variable, and the radiation [40]. The correlation coefficient ranges from 0 to 1 , and it works under the assumption of a Gaussian distribution of linear data.

Table 2. Descriptive statistics for the radiation values of the three zones: The arithmetic mean, the minimum radiation, and the maximum radiation (expressed in $\mathrm{kWh} / \mathrm{m}^{2}$ ).

\begin{tabular}{cccc}
\hline $\begin{array}{c}\text { Arithmetic } \\
\text { Mean/Minimum/Maximum }\end{array}$ & Zone 1 & Zone 2 & Zone 3 \\
\hline January & $1.083 / 0.003 / 2.039$ & $1.221 / 0.016 / 1.984$ & $1.299 / 0.007 / 2.020$ \\
February & $2.085 / 0.004 / 3.702$ & $2.337 / 0.025 / 3.645$ & $2.487 / 0.011 / 3.691$ \\
March & $3.277 / 0.005 / 5.141$ & $3.601 / 0.032 / 5.067$ & $3.902 / 0.014 / 5.119$ \\
April & $4.737 / 0.005 / 6.710$ & $5.154 / 0.030 / 6.632$ & $5.605 / 0.013 / 6.685$ \\
May & $6.034 / 0.005 / 8.078$ & $6.499 / 0.056 / 8.012$ & $7.084 / 0.120 / 8.061$ \\
June & $6.275 / 0.004 / 8.262$ & $6.737 / 0.052 / 8.202$ & $7.440 / 0.011 / 8.310$ \\
July & $6.284 / 0.003 / 8.331$ & $6.750 / 0.041 / 8.277$ & $7.366 / 0.009 / 8.291$ \\
August & $5.481 / 0.003 / 7.634$ & $5.941 / 0.019 / 7.583$ & $6.469 / 0.008 / 7.599$ \\
September & $4.052 / 0.004 / 6.304$ & $4.468 / 0.023 / 6.222$ & $5.605 / 0.013 / 6.685$ \\
October & $2.723 / 0.006 / 4.531$ & $3.028 / 0.034 / 4.456$ & $3.229 / 0.015 / 4.518$ \\
November & $1.590 / 0.003 / 3.113$ & $1.806 / 0.021 / 3.049$ & $1.911 / 0.009 / 3.092$ \\
December & $1.012 / 0.002 / 2.044$ & $1.161 / 0.015 / 1.995$ & $1.135 / 0.004 / 2.011$ \\
\hline
\end{tabular}

\section{Results}

The directional statistical analysis of the distribution of the monthly solar potential of rooftops in the city of Cáceres, in this study, was based on the vector distribution. Since vectors have a module and an azimuth, analyses from different perspectives could be performed. In this study, the module of the vector corresponds to the radiation value. Table 2 provides some descriptive statistics for the radiation values (expressed in units of $\mathrm{kWh} / \mathrm{m}^{2}$ ) of the three zones: The arithmetic mean, the minimum radiation, and the maximum radiation values received.

The values of the arithmetic means in zones 1,2 , and 3 were $3.77,4.06$, and $4.46 \mathrm{kWh} / \mathrm{m}^{2}$, respectively. The minimum value of the received radiation $\left(0.003 \mathrm{kWh} / \mathrm{m}^{2}\right)$ was found in zone 1 . A higher value was reached for zone $2\left(0.03 \mathrm{kWh} / \mathrm{m}^{2}\right)$, and a mean value of $0.01 \mathrm{kWh} / \mathrm{m}^{2}$ was found in zone 3 . These three cases show little variation among all of the months. Therefore, zone 1 , in the city center, received less radiation than the other two zones, and the differences in the average solar potential between the three studied zones reached $9 \%$ between zones 1 and 2, and between zones 2 and 3 , and $16 \%$ between zones 1 and 3 .

The maximum values of the arithmetic means and the maximum values of the radiation were obtained in July for zones 1 and 2, and in June for zone 3 . The maximum values of the arithmetic means in zones 1,2 , and 3 were $6.28,6.75$, and $7.44 \mathrm{kWh} / \mathrm{m}^{2}$, respectively, and the maximum values of the radiation were $8.33,8.27$, and $8.31 \mathrm{kWh} / \mathrm{m}^{2}$, respectively, with the same criteria. The minimum values 
of the arithmetic mean were obtained in December for zones $2\left(1.16 \mathrm{kWh} / \mathrm{m}^{2}\right)$ and $3\left(1.13 \mathrm{kWh} / \mathrm{m}^{2}\right)$, and in January for zone $1\left(1.08 \mathrm{kWh} / \mathrm{m}^{2}\right)$.

Other descriptive statistics revealed that all of the skewness coefficients were negative. Thus, the tail of the left side of the distribution was longer than that of the right side, especially in the month of July, as seen in Figure 3.

Figure 3 shows the histograms corresponding to July and December for the three studied areas. The same histograms were wrapped around for each year, thereby giving the values for the orientations in the two-variable circular histogram graphics (Figure 6).

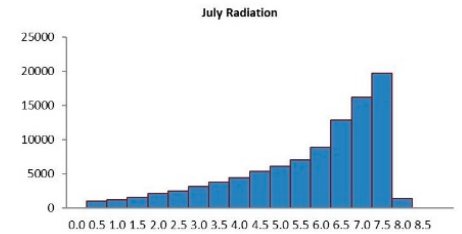

(a)

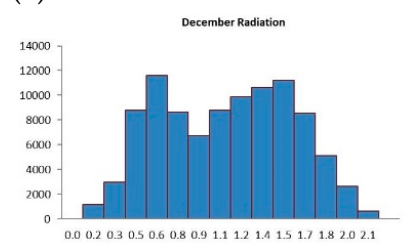

(a)

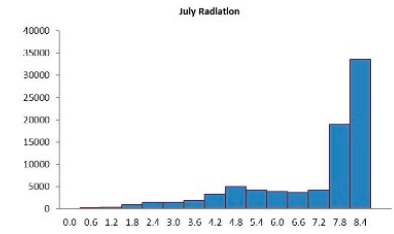

(b)

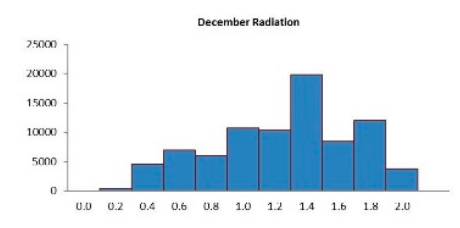

(b)

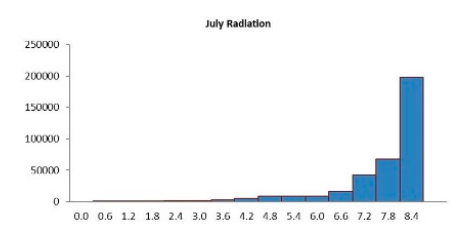

(c)

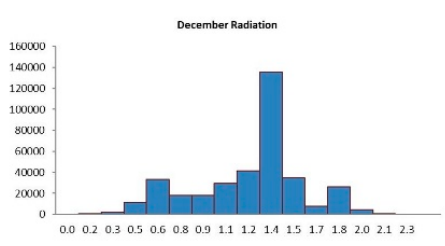

(c)

Figure 3. Histograms representing the radiation values in July and December in (a) zone 1, (b) zone 2, and (c) zone 3.

In the directional statistical analysis of the distribution of the monthly solar potential of rooftops in the city of Cáceres, in this study, the azimuth of the vector corresponded to the orientation value. Table 3 shows some of the circular statistics for the orientations in the three studied zones. The circular statistics employed herein were the mean azimuth, which represented the mean direction of the orientations, the circular variance, and the circular standard deviation, all of which were measurements of the dispersion. In contrast, the concentration parameter was a measure of the concentration of the data around a preferred orientation.

Table 3. Circular statistics for the values of the orientation for the three studied zones.

\begin{tabular}{cccc}
\hline & Zone 1 & Zone 2 & Zone 3 \\
\hline Mean azimuth & $306.24^{\circ}$ & $20.57^{\circ}$ & $218.12^{\circ}$ \\
Circular Variance & 0.97 & 0.97 & 0.98 \\
Circular std. Deviation & $154.53^{\circ}$ & $154.04^{\circ}$ & $163.86^{\circ}$ \\
Concentration Parameter & 0.05 & 0.05 & 0.03 \\
\hline
\end{tabular}

The mean azimuth was not a significant parameter because of the distribution of the data. The high values of the circular standard deviation revealed a large dispersion within the data. In addition, the circular variance ranged from 0 to 1 , where smaller values of the circular variance indicated that the data were more concentrated. The concentration parameter was very low in all cases $(0.05$, 0.05 , and 0.03 in zones 1,2 , and 3 , respectively); therefore, there was no preferred orientation in any case. The concentration parameter was 0 in a uniform distribution and was considered significant when greater than 2 . In any case, the data did not follow a uniform distribution according to the test of uniformity.

Figure 4 presents the values of the radiation as blue symbols measured clockwise from $0^{\circ}$ (north). In all cases, due to the large number of observations, each symbol represented multiple observations, but the proportionality of the figure was maintained. 
The circular graphics representing the orientation data show the distribution of the houses in relation to the urban framework.

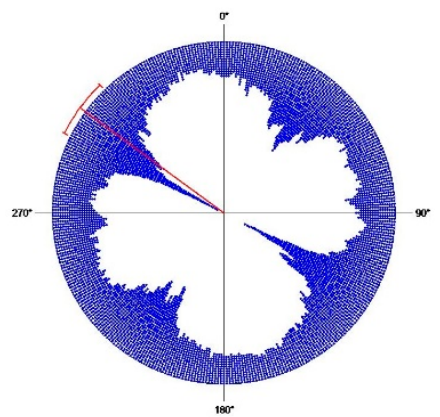

(a)

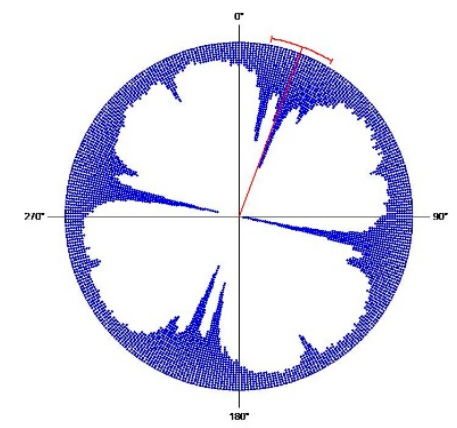

(b)

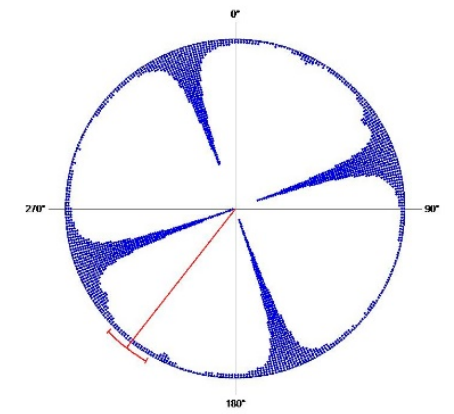

(c)

Figure 4. Raw orientation data in (a) zone 1, (b) zone 2, and (c) zone 3. The mean azimuth is plotted in red.

Zone 1 in the city center presented a more dispersed urban framework, as was reflected in the variation in the orientation. In zone 2, a new residential area on the outskirts of the city with a more regular framework, had a greater number of orientations in the two main directions. In zone 3, an industrial area, practically all buildings were located along two perpendicular directions.

Similar to the orientation, the slopes of the gables were also analyzed. Table 4 shows the same circular statistics used to explain the gable orientations.

Table 4. Circular statistics for the values of the slopes for the three zones.

\begin{tabular}{cccc}
\hline & Zone 1 & Zone 2 & Zone 3 \\
\hline Mean azimuth & $58.25^{\circ}$ & $61.03^{\circ}$ & $73.07^{\circ}$ \\
Circular Variance & 0.080 & 0.081 & 0.042 \\
Circular std. Deviation & $22.95^{\circ}$ & $23.51^{\circ}$ & $16.69^{\circ}$ \\
Concentration Parameter & 6.77 & 6.47 & 12.30 \\
\hline
\end{tabular}

Logically, all of the slopes ranged from 0 to $90^{\circ}$. The lowest mean azimuth was $58.25^{\circ}$ in the city center (zone 1), and the highest mean azimuth $\left(73.07^{\circ}\right)$ corresponded to the lower slope values in the industrial area (zone 3). Unlike the case of the orientations, the circular standard deviation values presented smaller results, while higher values were observed for the concentration parameter, thereby indicating the existence of a preferred orientation. The data were more concentrated around the mean azimuth, especially in zone 3, which exhibited a very high concentration parameter (12.30). The low values of the circular variance were in accordance with this hypothesis.

Figure 5 presents the values of the slope as blue symbols measured from the horizontal plane. In all of these cases, due to the large number of observations, each symbol represented multiple observations, but the proportionality of the figure was maintained. 


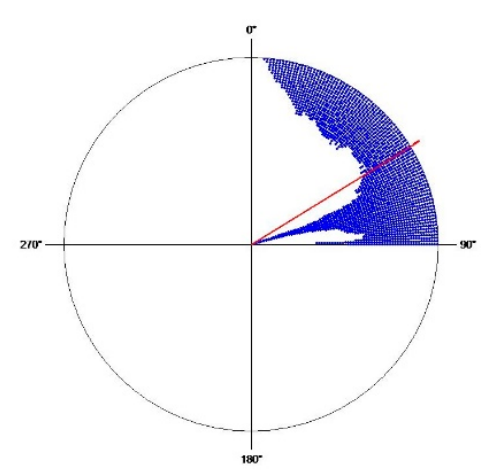

(a)

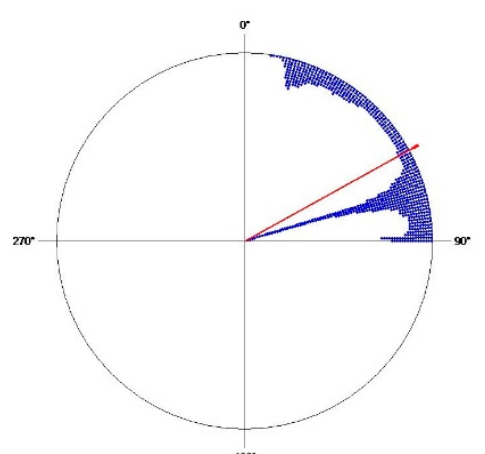

(b)

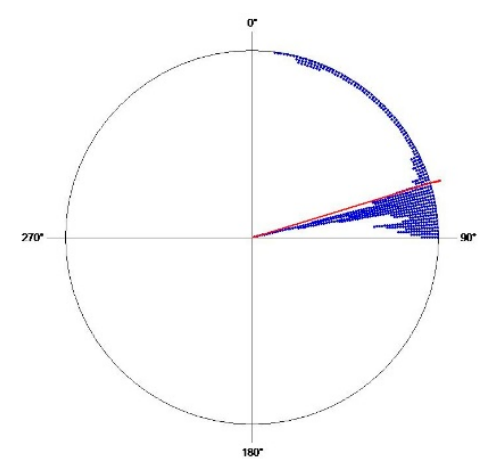

(c)

Figure 5. Raw slope data in (a) zone 1, (b) zone 2, and (c) zone 3. The mean azimuth is plotted in red.

The circular graphics representing the slope data of the rooftops were related to the typologies of the buildings. Zone 1, which contained older and heterogeneous types of buildings, presented a greater variety of slopes, whereas the industrial area (zone 3) had more concentrated and horizontal slope values, and zone 2 presented an intermediate situation between zones 1 and 3 .

The following discusses the joint analysis of the linear (radiation) and circular (orientation) data. The circular-linear correlation coefficient represented the correlation between both the orientation variable and the radiation variable, and ranged from 0 to 1 . Therefore, there was no negative correlation. Due to the low $p$-values in all cases, the null hypothesis of correlation between the two variables was rejected.

The two-variable circular histogram graphic (Figure 6) displays a histogram wrapped around the circle, where each section of the histogram is a $30^{\circ}$ wedge that depicts the number of observations (radiation values) falling within that portion of the range. Blue colors represent low radiation values, while red colors imply a higher level of received radiation.
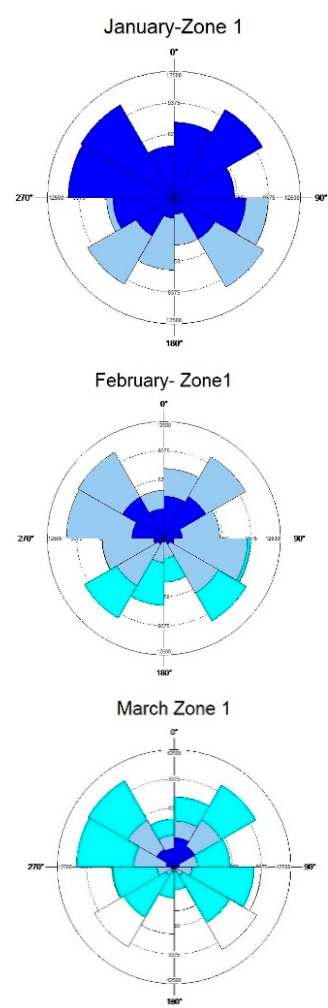
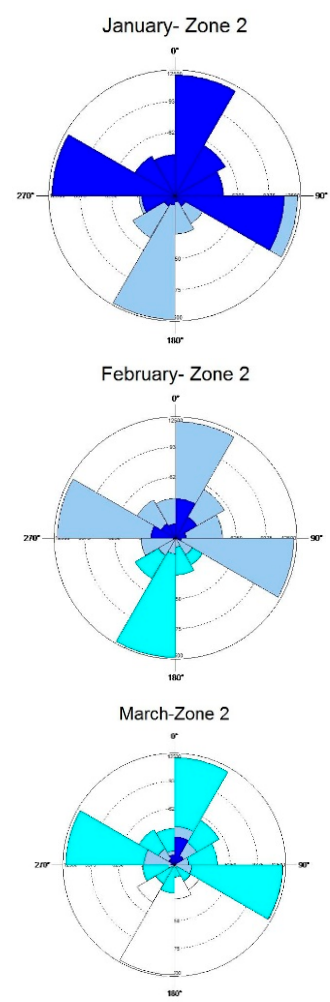

Figure 6. Cont.
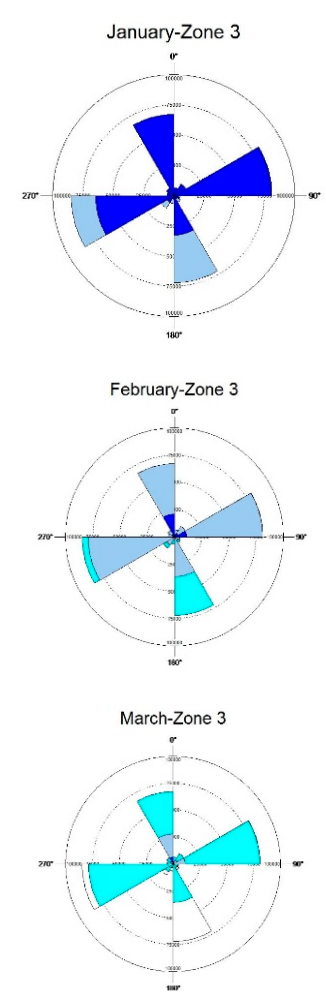

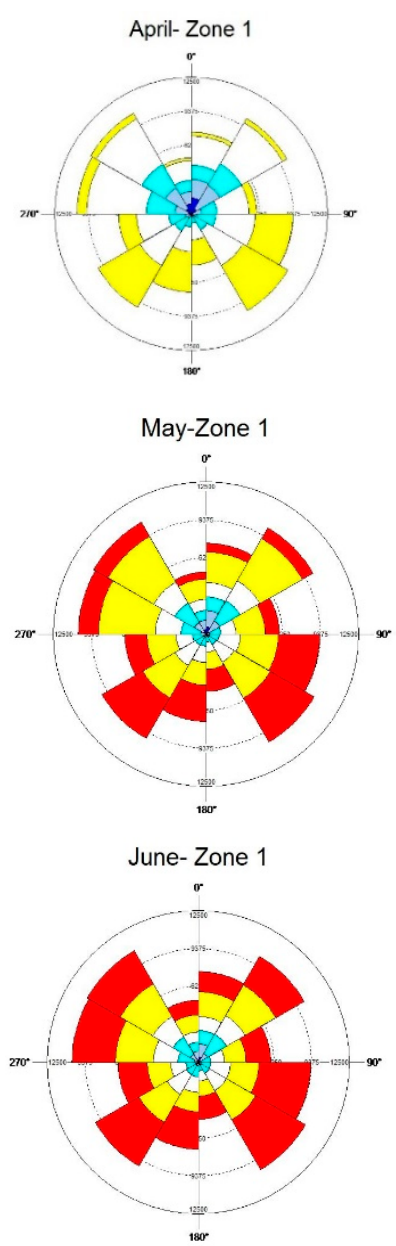

July-Zone 1

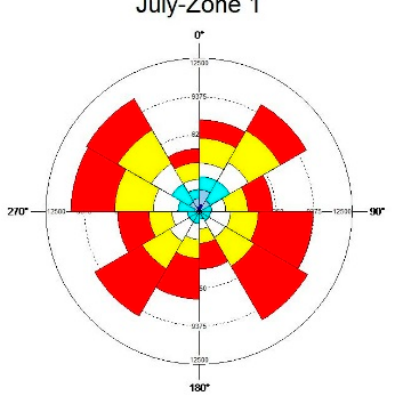

August-Zone 1

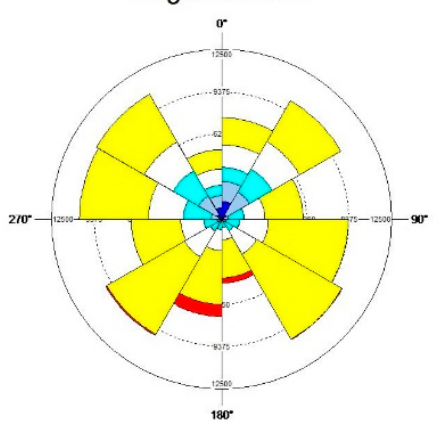

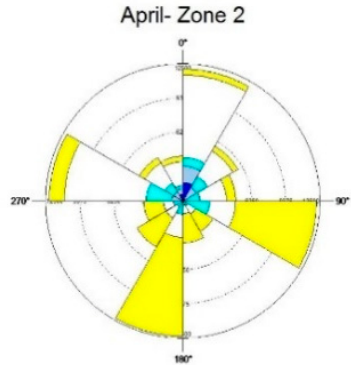

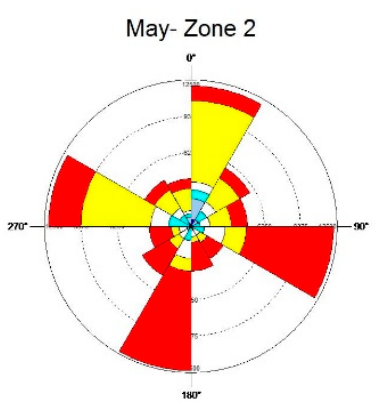

June-Zone 2

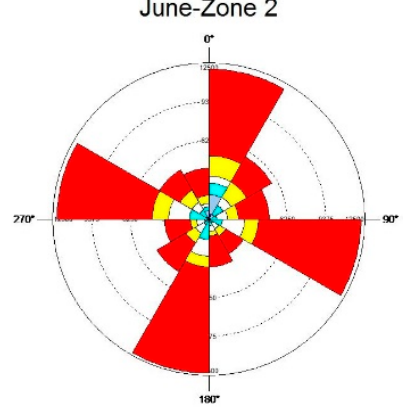

July-Zone 2

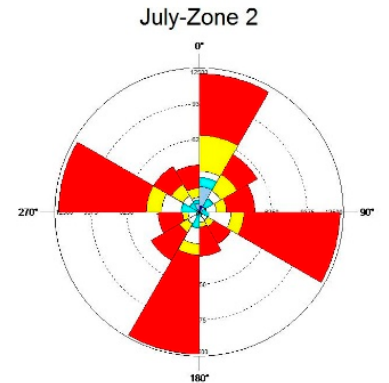

August-Zone 2

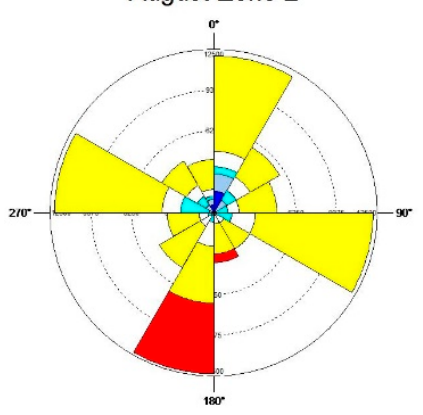

Figure 6. Cont.
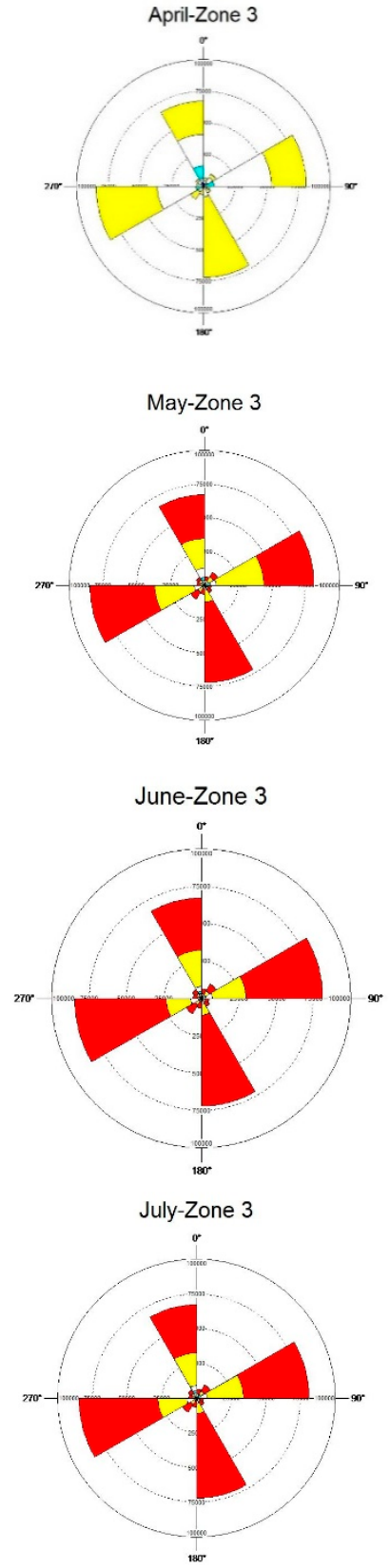

August-Zone 3

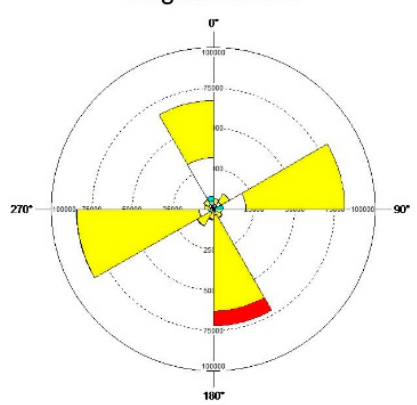



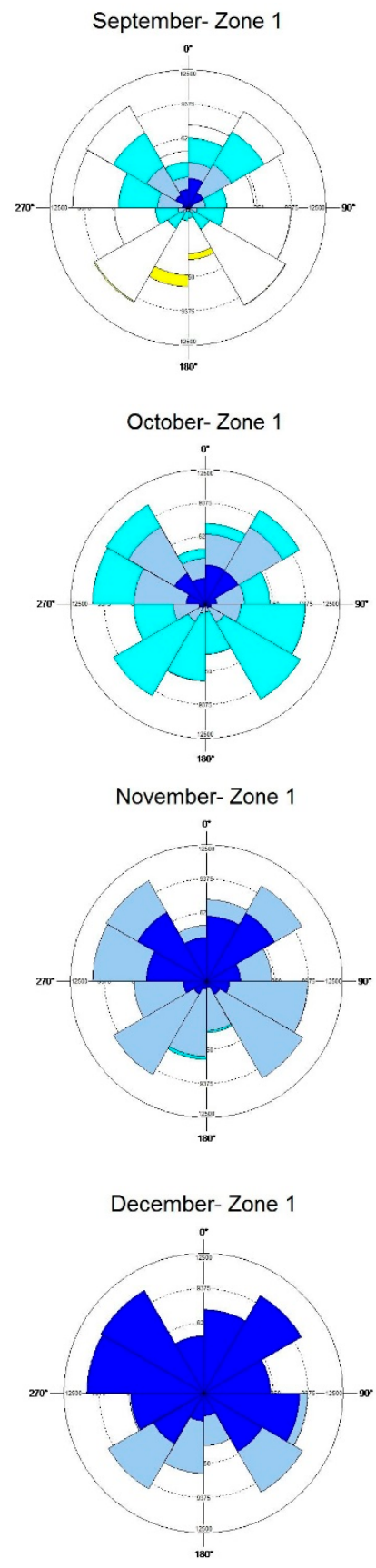
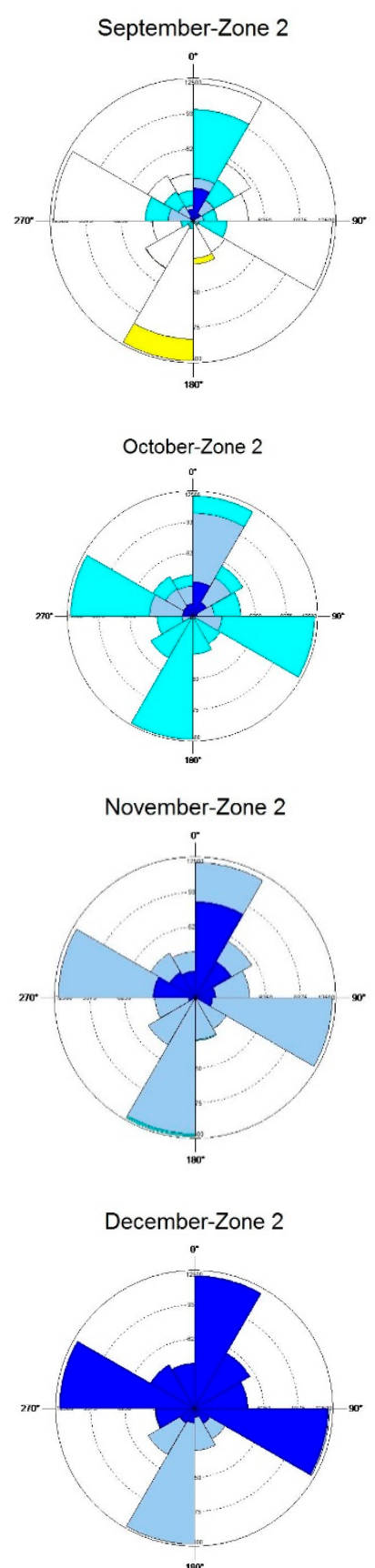
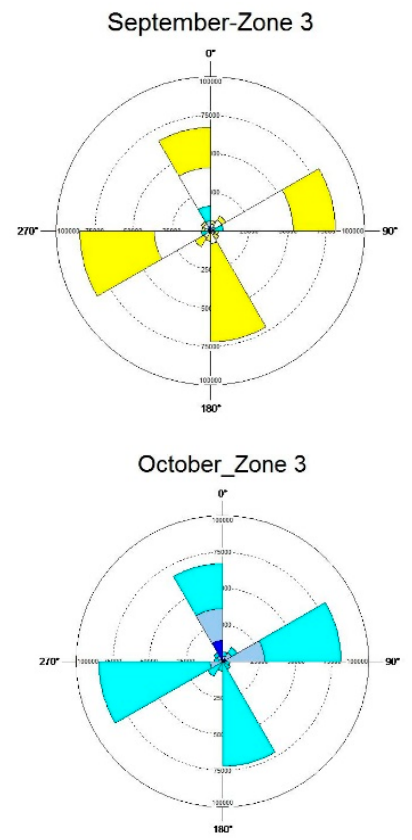

November-Zone 3

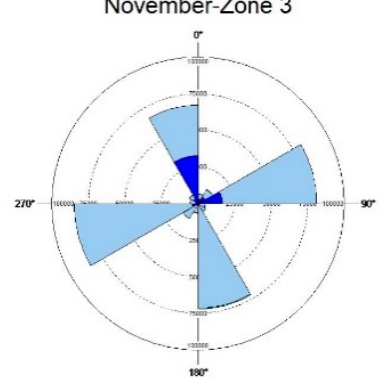

December-Zone 3

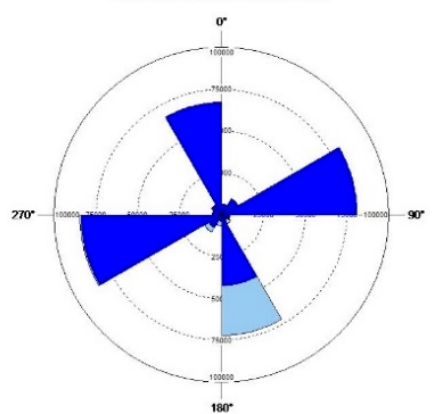

Figure 6. Two-variable circular histogram graphics of the three zones for every month of the year.

According to the two-variable circular histogram graphics in Figure 6, the roofs oriented towards the north received less radiation than those oriented towards the south, especially from September to March. In those months, the highest contrasts were located in zone 1, where the difference between the north and south orientations was approximately $52 \%$. In contrast, from May to July, the Sun was higher and the radiation values for the two orientations were relatively equal. In this way, the smallest differences were located in zone 3 , with only a $10 \%$ variation in the potential radiation between the north- and south-facing rooftops.

\section{Discussions}

The numerical and graphical results presented above allow a comparison of the radiation values, over an entire year, for different types of buildings in a medium-sized city in Spain. Using this type 
of statistical analysis could enable this methodology to be extrapolated to other cities with different urban frameworks and solar radiation potentials. This approach is; therefore, a useful tool to handle the challenges associated with climate change.

The circular graphics representing the slope data of the rooftops are related to the typologies of the buildings in the three different zones. According to the two-variable circular histogram graphics, the roofs oriented towards the north received less radiation than those oriented towards the south, especially from September to March. In contrast, from May to July, the Sun was higher, and the radiation received by the rooftops oriented in both directions were relatively similar. These results confirm those obtained by [46], who determined that the solar radiation varies with the geographic latitude, that the shortest period faces the direct sunshine, and that the solar radiation falling on the north-facing vertical plane is the lowest. More specifically, other studies conducted at similar geographic latitudes, such as [47], confirmed that the optimal building rooftop and façade orientation for the installation of a PV system faces the south when implementing solar urban planning.

In light of our results, a $10 \%$ variation in the potential radiation between north- and south-facing rooftops was found in zone 3 for the summer months. This result is in accordance with the findings of [13], who obtained a $15 \%$ higher annual solar potential for south- and southwest-facing roofs in working areas at approximately the same latitude. In contrast, zone 1 exhibited differences of approximately $52 \%$ between the north and south orientations, from the autumn to the spring seasons. These variations confirm the achievements of [42], who concluded that the morphology of each district is crucial for determining the solar potential.

Nevertheless, other works such as [25] found a direct relation between the orientation and the annual solar irradiation in 16 studied neighborhoods, due to the high dispersion of orientations for the studied buildings. They concluded that the building density has a larger impact on the solar radiation than the building orientation. Ref. [42] also confirmed a difference of $15 \%$ in the annual solar potential between two different neighborhoods. All of these findings support the differences reached in the average solar potential between the three studied zones, from $9 \%$ between zones 1 and 2 and between zones 2 and 3, to $16 \%$ between zones 1 and 3 . In addition, the precision of the monthly evaluation in each zone can be extended to detect the months with the highest differences (November and December), and those with only slight differences in the radiation values among the different zones (June and July).

Circular graphics representing orientation data suitably represented the distribution of buildings within an urban framework. In this way, ref. [48] established that the impact of the geometry of a city on the solar energy potential was significant (up to twice as much as the impacts of other factors). Regarding the urban characteristics, the differences in the solar radiation found in the three different neighborhoods are also in accordance with the findings of studies such as [42], in which the organizations of streets, buildings, and other urban elements had high impacts on the obtained results. However, none of the abovementioned studies considered the monthly radiation and instead focused only on the annual solar irradiation.

Finally, regarding the slope analysis, the inclusion of the real inclinations of rooftops improved upon the results obtained by works such as [41] that were limited by the use of parametric models and by the consideration of horizontal roofs.

\section{Conclusions}

In this work, a directional statistical analysis of the distribution of the monthly solar potential of rooftops in the city of Cáceres in relation to the orientations and slopes of the rooftops was performed in three different neighborhoods. The precision of the evaluation is attributable to not only the resolution of the rooftops but also the monthly disaggregation of the results. In this sense, two residential areas, one in the city center and one on the outskirts of the city, and an industrial zone, all of which possessed different urban morphologies, were evaluated by examining more than 500,000 gables that precisely defined the rooftops. 
The three dissimilar urban morphologies resulted in different solar potential values, reaching up to $16 \%$. Additionally, the monthly disaggregation of data provided the ability to detect the highest differences between the north and south orientations (52\%) located in the city center, which exhibited irregular streets and different types of buildings, taking place from September to March.

The achievements of the present work reveal more in-depth information regarding the urban characteristics that more effectively capture solar energy. The proposed analysis could also be extrapolated to urban planning for the design of more sustainable cities to face the challenges associated with climate change.

Author Contributions: M.-E.P., M.P., and E.Q. conceived and designed the experiments; M.-E.P., M.P., and E.Q. performed the experiments; M.-E.P., M.P., and E.Q. analyzed the data; M.-E.P., M.P., and E.Q. contributed reagents, materials, and analysis tools; and M.-E.P., M.P., and E.Q., wrote the paper.

Funding: This publication was supported by the Government of Extremadura (Spain) (Project GR15129) and was co-funded by the European Regional Development Fund.

Acknowledgments: The founding sponsors had no role in the design of the study, in the collection, analyses, or interpretation of the data, in the writing of the manuscript, or in the decision to publish the results. The authors thank both the Local Council GIS Service of Cáceres and the Spanish National Meteorological Agency AEMET for providing the data and for their continued assistance. They also would like to thank the editors and the anonymous reviewers for their careful revision of our manuscript and for their comments and suggestions, which greatly helped to improve the quality of the work.

Conflicts of Interest: The authors declare no conflicts of interest.

\section{References}

1. Lacal Arantegui, R.; Jäger-Waldau, A. Photovoltaics and wind status in the European Union after the Paris Agreement. Renew. Sustain. Energy Rev. 2018, 81, 2460-2471. [CrossRef]

2. Biyik, E.; Araz, M.; Hepbasli, A.; Shahrestani, M.; Yao, R.; Shao, L.; Essah, E.; Oliveira, A.C.; del Caño, T.; Rico, E.; et al. A key review of building integrated photovoltaic (BIPV) systems. JESTECH 2017, 20, 833-858. [CrossRef]

3. Hoggett, R. Technology scale and supply chains in a secure, affordable and low carbon energy transition. Appl. Energy 2014, 123, 296-306. [CrossRef]

4. Bazán, J.; Rieradevall, J.; Gabarrell, X.; Vázquez-Rowe, I. Low-carbon electricity production through the implementation of photovoltaic panels in rooftops in urban environments: A case study for three cities in Peru. Sci. Total Environ. 2018, 622-623, 1448-1462. [CrossRef] [PubMed]

5. Katiyar, A.K.; Pandey, C.K. A Review of Solar Radiation Models. Part I. J. Renew. Energy 2013. [CrossRef]

6. Seo, D. Development of a Universal Model for Predicting Hourly Solar Radiation-Application: Evaluation of an Optimal Day Lighting Controller. Ph.D. Thesis, University of Colorado, Boulder, CO, USA, 2010.

7. Marcel, Š.; Jaroslav, H. A New GIS-based Solar Radiation Model and Its Application to Photovoltaic Assessments. Trans. GIS 2004, 8, 175-190.

8. Fu, P.; Rich, P.M. Design and implementation of the Solar Analyst: An ArcView extension for modeling solar radiation at landscape scales. In Proceedings of the Nineteenth Annual ESRI User Conference, San Diego, CA, USA, 26-30 July 1999; pp. 1-31.

9. Bezir, N.C.; Akkurt, I.; Özek, N. The Development of a Computer Program for Estimating Solar Radiation. Energy Sour. Part A-Recover. Util. Environ. Eff. 2010, 32, 995-1003. [CrossRef]

10. Bayrakçı, H.C.; Demircan, C.; Keçebaş, A. The development of empirical models for estimating global solar radiation on horizontal surface: A case study. Renew. Sustain. Energy Rev. 2018, 81, 2771-2782. [CrossRef]

11. Nguyen, H.T.M.; Pearce, J. Automated quantification of solar photovoltaic potential in cities. Int. Rev. Spat. Plan. Sustain. Dev. 2013, 1, 49-60. [CrossRef]

12. Singh, R.; Banerjee, R. Estimation of rooftop solar photovoltaic potential of a city. Sol. Energy 2015, 115, 589-602. [CrossRef]

13. Brito, M.C.; Gomes, N.; Santos, T.; Tenedório, J.A. Photovoltaic potential in a Lisbon suburb using LiDAR data. Sol. Energy 2012, 86, 283-288. [CrossRef]

14. Nguyen, H.T.; Pearce, J.M. Incorporating shading losses in solar photovoltaic potential assessment at the municipal scale. Sol. Energy 2012, 86, 1245-1260. [CrossRef] 
15. La Gennusa, M.; Lascari, G.; Rizzo, G.; Scaccianoce, G.; Sorrentino, G. A model for predicting the potential diffusion of solar energy systems in complex urban environments. Energy Policy 2011, 39, 5335-5343. [CrossRef]

16. Lukač, N.; Žalik, B. GPU-based roofs' solar potential estimation using LiDAR data. Comput. Geosci. 2013, 52, 34-41. [CrossRef]

17. Bergamasco, L.; Asinari, P. Scalable methodology for the photovoltaic solar energy potential assessment based on available roof surface area: Further improvements by ortho-image analysis and application to Turin (Italy). Sol. Energy 2011, 85, 2741-2756. [CrossRef]

18. Melo, E.G.; Almeida, M.P.; Zilles, R.; Grimoni, J.A.B. Using a shading matrix to estimate the shading factor and the irradiation in a three-dimensional model of a receiving surface in an urban environment. Sol. Energy 2013, 92, 15-25. [CrossRef]

19. Brito, M.C.; Freitas, S.; Guimarães, S.; Catita, C.; Redweik, P. The importance of facades for the solar PV potential of a Mediterranean city using LiDAR data. Renew Energy 2017, 111, 85-94. [CrossRef]

20. Martínez-Rubio, A.; Sanz-Adan, F.; Santamaría-Peña, J.; Martínez, A. Evaluating solar irradiance over facades in high building cities, based on LiDAR technology. Appl. Energy 2016, 183, 133-147. [CrossRef]

21. Quirós, E.; Pozo, M.; Ceballos, J. Solar potential of rooftops in Cáceres city, Spain. J. Maps 2018, 14, 44-51. [CrossRef]

22. Bizjak, M.; Žalik, B.; Štumberger, G.; Lukač, N. Estimation and optimisation of buildings' thermal load using LiDAR data. Build Environ. 2018, 128, 12-21. [CrossRef]

23. Lingfors, D.; Bright, J.M.; Engerer, N.A.; Ahlberg, J.; Killinger, S.; Widén, J. Comparing the capability of lowand high-resolution LiDAR data with application to solar resource assessment, roof type classification and shading analysis. Appl. Energy 2017, 205, 1216-1230. [CrossRef]

24. Hachem, C.; Athienitis, A.; Fazio, P. Investigation of solar potential of housing units in different neighborhood designs. Energy Build. 2011, 43, 2262-2273. [CrossRef]

25. Mohajeri, N.; Upadhyay, G.; Gudmundsson, A.; Assouline, D.; Kämpf, J.; Scartezzini, J.-L. Effects of urban compactness on solar energy potential. Renew. Energy 2016, 93, 469-482. [CrossRef]

26. Takebayashi, H.; Ishii, E.; Moriyama, M.; Sakaki, A.; Nakajima, S.; Ueda, H. Study to examine the potential for solar energy utilization based on the relationship between urban morphology and solar radiation gain on building rooftops and wall surfaces. Sol. Energy 2015, 119, 362-369. [CrossRef]

27. Sarralde, J.J.; Quinn, D.J.; Wiesmann, D.; Steemers, K. Solar energy and urban morphology: Scenarios for increasing the renewable energy potential of neighbourhoods in London. Renew. Energy 2015, 73, 10-17. [CrossRef]

28. Ling, C.S.; Ahmad, M.H.; Ossen, D.R. The Effect of Geometric Shape and Building Orientation on Minimising Solar Insolation on High-Rise Buildings in Hot Humid Climate. J. Constr. Dev. Ctries. 2007, 12, 27-38.

29. Hartner, M.; Ortner, A.; Hiesl, A.; Haas, R. East to west-The optimal tilt angle and orientation of photovoltaic panels from an electricity system perspective. Appl. Energy 2015, 160, 94-107. [CrossRef]

30. Chen, C.; Li, Y.; Li, N.; Wei, S.; Yang, F.; Ling, H.; Yu, N.; Han, F. A computational model to determine the optimal orientation for solar greenhouses located at different latitudes in China. Sol. Energy 2018, 165, 19-26. [CrossRef]

31. Skeiker, K. Optimum tilt angle and orientation for solar collectors in Syria. Energy Convers. Manag. 2009, 50, 2439-2448. [CrossRef]

32. Batschelet, E. Circular Statistics in Biology; Academic Press: London, UK, 1981; p. 371.

33. Rodríguez, P.G.; Polo, M.E.; Cuartero, A.; Felicísimo, Á.M.; Ruiz-Cuetos, J.C. VecStatGraphs2D, A Tool for the Analysis of Two-Dimensional Vector Data: An Example Using QuikSCAT Ocean Winds. IEEE Geosci. Remote Sens. Lett. 2014, 11, 921-925. [CrossRef]

34. Corcoran, J.; Chhetri, P.; Stimson, R. Using circular statistics to explore the geography of the journey to work. Pap. Reg. Sci. 2009, 88, 119-132. [CrossRef]

35. Cuartero, A.; Felicísimo, Á.M.; Polo, M.E.; Caro, A.; Rodríguez, P.G. Positional accuracy analysis of satellite imagery by circular statistics. Photogramm. Eng. Remote Sens. 2010, 76, 1275-1286. [CrossRef]

36. Quiros, E.; Polo, M.E.; Felicisimo, A.M. Detection and Labeling of Sensitive Areas in Hydrological Cartography Using Vector Statistics. IEEE Trans. Geosci. Remote Sens. 2016, 54, 189-196. [CrossRef]

37. Polo, M.E.; Felicísimo, Á.M. Full Positional Accuracy Analysis of Spatial Data by Means of Circular Statistics. Trans. GIS 2010, 14, 421-434. [CrossRef] 
38. Fisher, N.I. Statistical Analysis of Circular Data, 2nd ed.; Cambridge University Press: Cambridge, UK, 1995; p. 277.

39. Jammalamadaka, S.R.; SenGupta, A. Topics in Circular Statistics; World Scientific Publishing: Singapore, 2001; Volume 5, p. 322.

40. Mardia, K.V.; Jupp, P.E. Directional Statistics; Wiley: Chichester, UK, 2000; p. 414.

41. Amado, M.; Poggi, F. Solar Urban Planning: A Parametric Approach. Energy Proced. 2014, 48, 1539-1548. [CrossRef]

42. Montavon, M.; Scartezzini, J.L.; Compagnon, R. Comparison of the solar energy utilisation potential of different urban environments. In Proceedings of the PLEA 2004 Conference, Eindhoven, The Netherlands, 19-21 September 2004.

43. Service, K.C. Oriana for Windows, 4.02; Kovach Computing Service: Pentraeth, Wales, UK, 2012.

44. $R$ Core Team R: A Language and Environment for Statistical Computing; R Foundation for Statistical Computing: Vienna, Austria, 2017.

45. IBM SPSS Statistics; 22. IBM Corp: New York, NY, USA, 2013. Available online: https://www.ibm.com/ analytics/es/es/ (accessed on 18 October 2018).

46. Li, D.H.W.; Lam, T.N.T. Determining the Optimum Tilt Angle and Orientation for Solar Energy Collection Based on Measured Solar Radiance Data. Int. J. Photoenergy 2007. [CrossRef]

47. Amado, M.; Poggi, F. Towards Solar Urban Planning: A New Step for Better Energy Performance. Energy Proced. 2012, 30, 1261-1273. [CrossRef]

48. Kanters, J.; Horvat, M. Solar Energy as a Design Parameter in Urban Planning. Energy Proced. 2012, 30, 1143-1152. [CrossRef]

(C) 2018 by the authors. Licensee MDPI, Basel, Switzerland. This article is an open access article distributed under the terms and conditions of the Creative Commons Attribution (CC BY) license (http:/ / creativecommons.org/licenses/by/4.0/). 